\title{
Correction to: "Clinic-Based Behavioral Skills Training to Teach Caregivers Adaptive Skill Interventions for Children with Autism"
}

\author{
Leslie Neely $^{1} \cdot$ Felicia Castro-Villarreal $^{1} \cdot$ Ee Rea Hong ${ }^{2} \cdot$ Kelly Ponce $^{1} \cdot$ Stephanie Gerow $^{3}$
}

Published online: 30 January 2021

(C) Springer Nature Switzerland AG 2021

\section{Correction to: "Advances in Neurodevelopmental Disorders" https://doi.org/10.1007/s41252-020-00166-7}

During revisions, the author requested to upload the data set to a repository. The author identified dryad as the repository and had set up the account, uploaded the data, and had it ready to publish contingent upon publication of the article. When the article was published, the author was informed that there was a fee associated with this repository. However, there is no funding associated with this research to facilitate this request.

The author was able to upload the data set to their UTSA repository (link: https://urldefense.proofpoint.com/v2/url?u=https3A_rrpress.utsa.edu_handle_20.500.12588_196\&d= D w I G a Q \& c $=$ vh 6 F g F nd u e j N P P P D $0 \mathrm{fl}$ y R a S f Z y $8 \mathrm{C} \mathrm{W} \mathrm{b} \mathrm{W} \mathrm{n} \mathrm{I} \mathrm{f} 4$ X J h S q x 8 \& r $=$ dpWkPNJOoNfEIfllxvOgux1lgUrTmwHXrbFJH3dH-fytHaT_ y4zvcSdtuIQi94x0\&m=x9SMOb42YUE0m1Zat5H1
G 3 O 65 kqj 8 V Z bo D B eN 8 o k y F s \& s = 1 T L k k l cnF7nxZXrs3k4D6L5D6Jg63I84AlwYLPXpQ4\&e=).

The two areas in the proof where the new link needs to be updated:

1. Pg. 9 - Should read "The raw data corresponding to Fig. 1 is available via online repository (Neely et al., 2020)

2. Pg. 14 - Replace "Dryad, Dataset. https://doi.org/10.5061/ dryad.qfttdz0dn." with "UTSA Roadrunner Research Press, Dataset. https://rrpress.utsa.edu/handle/20.500.12588/196."

Thus, this erratum is presented to fix the error.

The original article has been corrected.

Publisher's Note Springer Nature remains neutral with regard to jurisdictional claims in published maps and institutional affiliations.

The online version of the original article can be found at https://doi.org/ $10.1007 / \mathrm{s} 41252-020-00166-7$

Leslie Neely

leslie.neely@utsa.edu

1 Department of Educational Psychology, The University of Texas at San Antonio, San Antonio, TX 78249, USA

2 Baekseok University, Cheonan, Republic of Korea

3 Baylor University, Waco, TX, USA 